\title{
Reference and Pragmatic Identification
}

\author{
Douglas E. Appelt \\ Artificial Intelligence Center \\ Center for the Study of Language and Information \\ SRI International
}

\section{Identification Constraints.}

Kronfeld [9] has presented the outline of a theory of referring that provides an excellent framework for explaining how referring actions operate in various contexts. However, it also raises some important questions, one of which I will examine in greater detail in this paper. This question is "Where do identification constraints come from?"

According to Kronfeld's model, the literal goal of a referring action is to make it mutually believed that "identification" of some entity is required. This idea is similar to that advanced by Cohen $[2,3]$, who argues in favor of analyzing referring as the illocutionary act of requesting as opposed to a propositional act, whereas Kronfeld's model retains aspects of the propositional act analysis. Regardless of the details of the proposal adopted, it does little good to say merely that a referring action requires identification of the referent, because the precise facts that must be known for a hearer to say that he has identified a referent is different in practically every case.

In the model under consideration, an individual is represented to an agent by an individuating set of terms, each believed to denote the individual. The ultimate goal of a referring action is to induce the hearer to identify a subset of one of his individuating sets that satisfies a number of identification constraints. The speaker and hearer must mutually know what the relevant identification constraints are in the current situation. Given this general theory of referring, the key problem becomes the explanation of how the speaker and hearer can agree on what identification constraints are currently applicable.

The point of this paper is that identification constraints come from a variety of sources, including knowledge about actions, general world knowledge, particular facts about the situation at hand, the semantic content of the referring expression, and principles of discourse. Each of these is an important area of analysis in its own right. I shall suggest how the various aspects of reference addressed by the members of the TINLAP-3 reference panel fit together under this general framework. 


\section{Constraints from World Knowledge}

Goodman [6] states that "Reference identification is a search process where a listener looks for something in the world that satisfies a speaker's uttered description." One can argue that this definition is too restrictive because it does not apply to situations in which an epistemological notion of identification is inappropriate. However, for task oriented dialogues such as those of Goodman's protocols, it is correct. The speaker and hearer are cooperating on a task that involves physical manipulation of assorted parts and tools. A reasonable theory of action would imply that physical manipulation of objects requires perception of the objects by the agent, and such a theory would be mutually believed by all agents. Therefore, the requirement that the individuating set contain a term resulting from some perceptual action would apply to nearly every reference to material objects in this domain.

Goodman's research is centered primarily on the problem of satisfying the referring request by carrying out the identification plan. Some of the bizarre referring expressions obtained from Goodman's protocols [5] are quite reasonable from the standpoint of achieving the literal referring goals. When the speaker used the referring expression "the champagne top sort of looking bottom" to refer to the tube base of the water pump, it is clear that (1) he interided the hearer to perceive the part, because he was asking the hearer to manipulate it, and (2) the referring description, consisting of perceptual descriptors, suggests a plan of visually observing objects in the domain and comparing their characteristics to those indicated by the description. The hearer in Goodman's protocol was unable to identify the intended object given this odd description, which demonstrates the need for the speaker to take both the satisfaction plan as well as the literal goals into account when planning a referring expression.

\section{Constraints from Definiteness}

The use of a definite determiner in a referring expression introduces an additional constraint on the hearer's individuating set: the individuating set must exist at the time of the utterance, or it must be implicitly associated [4] with an existing individuating set, i.e. its existence can be inferred from its association with an existing entity. This constraint prevents the hearer from creating an individuating set containing only the speaker's referring expression, which would amount to hypothesizing an entirely new entity. There is no such constraint associated with the indefinite determiner, which leaves the hearer free to hypothesize new individuating sets in the absence of any other prohibitive constraints. 
Other constraints may be brought to bear on the individuating set as well. For example, if the speaker is requesting the hearer to manipulate the entity introduced with the indefinite article, a perceptual term must be part of the individuating set. For example, if the speaker says "There is a philips screwdriver in the toolbox that you can use to fix the pump," the hearer's individuating set must contain a perceptual term denoting some existing object that he can perceive. Contextual information can sometimes be strong enough to imply a very strict criterion for referent identification. For example, at a testimonial dinner honoring John, a speaker says "We are gathered here to honor a gentleman and a scholar." The hearer must know there is only one person honored at the banquet, and that is John. Therefore, the individuating set specified must be the same as his individuating set for John, except that it must contain the descriptors "gentleman" and. "scholar." The speaker has exploited the overwhelming contextual influence to produce an expression that performs both informing and referring functions. This strategy is called action subsumption [1]. Dahl [4] discusses several more complex situations in which the use of an indefinite noun phrase is not permitted to introduce new individuals.

\section{Constraints from Discourse}

A particularly interesting set of referring expressions are those that also have anaphoric connection to the preceeding discourse. Not all anaphoric expressions are referring expressions. For example, in the sentence "No AI researcher will admit that he is wrong." neither the pronoun he nor its antecedent is a referring expression. However, pronouns and anaphoric definite noun phrases are frequently referring expressions. Because pronouns must refer anaphorically (or to some very salient object in the context), the identification constraints that apply to a pronominal referring expression are simple to state: The active individuating set must contain a term from the individuating set intended by a previous reference to the same individual, with gender and number providing additional constraints on the possible referent.

It is not so simple, however, to state how the satisfaction of the anaphoric identification request takes place. Much research in recent years has been devoted to this problem, including (to mention only a few instances) recent work on discourse context and centering by Grosz, Joshi, Sidner, and Weinstein [7,8], and Webber $[10,11]$. It is far beyond the

scope of this paper to discuss this work here, or to add anything to it. The reader should bear in mind that the principles of centering and the construction of discourse models, event/situation structures, etc. are all mutually known to the speaker and hearer in a dialogue. The speaker takes these principles into account when reasoning about how the 
hearer can formulate a plan to identify the referent of an anaphoric referring expression.

Identification constraints from multiple sources are necessary to explain changes that may take place in the identification constraints applicable to different instances of coreferential expressions. Consider the following sequence of utterances:

I am looking for a screwdriver.

It has a green handle.

Have you seen it recently?

In the first sentence, no constraints apply to the identification of the referent of "a screwdriver." The hearer therefore constructs a new individuating set to represent it. In understanding the second sentence, the hearer uses the centering algorithm to determine that the intended individuating set for the pronoun is the same as the one intended in the first sentence. The fact that the hearer intends the same individuating set for the pronoun in the third sentence can also be determined from the centering algorithm. However, the fact that the hearer is asked if he has seen the object implies that an additional identification constraint must be imposed on the individuating set at that time: the referent must be perceptually identified. A cooperative speaker must reason that the hearer has enough knowledge to satisfy the identification request before deciding that "it" constitutes an appropriate referring expression.

\section{Conclusion}

If referring is to be regarded as an action that requires the hearer to pragmatically identify the referent of a description, then it is important to describe how it is that the speaker and hearer know what pragmatic identification means in a given situation. This paper suggests that the situation dependent meaning of identification follows from general world knowledge, the syntactic and semantic structure of the referring expression itself, and principles of discourse anaphora resolution. This is by no means an exhaustive analysis of the ways in which identification conditions are recognized, but is rather intended to provide the first steps toward the analysis of reference in a framework that links the results several diverse research programs.

\section{Acknowledgements}

This research was supported by the National Science Foundation under grant DCR8407238. The author is grateful to Amichai Kronfeld for comments on the draft of this 
article, and for stimulating discussion of these and related issues.

\section{References}

[1] Douglas E. Appelt. Planning English Sentences. Cambridge University Press, Cambridge, England, 1985.

[2] Philip R. Cohen. The need for identification as a planned action. In Proceedings of the Seventh International Joint Conference on Artificial Intelligence, pages 31-36, 1981.

[3] Philip R. Cohen. Referring as requesting. In Proceedings of the Tenth International Conference on Computational Linguistics, pages 207-211, 1984.

[4] Deborah Dahl. Determiners, entities, and contexts. In this volume.

[5] Brad Goodman. Repairing reference identification failures by relaxation. In Proceedings of the 23rd Annual Meeting, pages 204-217, Association for Computational Linguistics, 1985.

[6] Bradly A. Goodman. Reference and reference failures. In this volume.

[7] Barbara J. Grosz, A. Joshi, and S. Weinstein. Providing a unified account of definite noun phrases in discourse. In Proceedings of the Twenty-first Annual Meeting, pages 44-50, Association for Computational Linguistics, 1983.

[8] Barbara J. Grosz and Candace L. Sidner. Discourse structure and the proper treatment of interruptions. In Proceedings of the Ninth International Joint Conference on Artificial Intelligence, pages 832-839, 1985.

[9] Amichai Kronfeld. Goals of referring acts. In this volume.

[10] Bonnie L. Webber. Description formation and discourse model synthesis. In Proceedings of TINLAP-2, pages 42-50, 1978.

[11] Bonnie L. Webber. Event reference. In this volume. 